\title{
Randomized and Deterministic Algorithms for Network Coding Problems in Wireless Networks
}

\author{
Zoltán Királya, ${ }^{\mathrm{a}, \mathrm{e}}$ Erika R. Kovács ${ }^{\mathrm{b}}$ \\ ${ }^{a}$ Department of Computer Science, Eötvös Loránd University, Budapest, Hungary \\ ${ }^{b}$ Department of Operations Research, Eötvös Loránd University, Budapest, Hungary \\ ${ }^{c}$ MTA-ELTE Egerváry Research Group
}

\begin{abstract}
Network coding is a method for information transmission in a network, based on the idea of enabling internal nodes to forward a function of the incoming messages, typically a linear combination. In this paper we discuss generalizations of the network coding problem with additional constraints on the coding functions called network code completion problem, NCCP. We give both randomized and deterministic algorithms for maximum throughput-achieving network code construction for the NCCP in the multicast case. We also introduce the related problem of fixable pairs, investigating when a certain subset of coding coefficients in the linear combination functions can be fixed to arbitrary non-zero values such that the network code can always be completed to achieve maximum throughput. We give a sufficient condition for a set of coding coefficients to be fixable. For both problems we present applications in different wireless and heterogenous network models.
\end{abstract}

\section{Introduction}

Network coding is a fairly new area on the boundary of information and graph theory, initiated by the seminal paper of Ahlswede et al.[1]. In contrast to a classical source-terminal encoding-decoding information transmission scheme, intermediate nodes of the network are enabled as well to perform coding. Properly chosen coding functions may increase throughput, security and reliability of a network [2]. Most algorithms apply linear network codes, when intermediate nodes forward a linear combination of the incoming messages. The coefficients in these linear combinations are called coding coefficients. In this paper, we investigate various linear network coding problems with partially predetermined coding coefficients. The first version of this problem, called deterministic network coding, was introduced by Harvey, Karger and Murota [3]. They reduced both the unicast and multicast cases to matrix completion. In order to avoid

Email addresses: kiraly@cs.elte.hu (Zoltán Király), koverika@cs.elte.hu (Erika R. Kovács) 
confusion of 'deterministic algorithm' and 'deterministic network coding problem' we call the latter the network code completion problem (NCCP) throughout the paper. In [4], Fragouli and Ebrahimi showed that the NCCP has an application for a Gaussian relay network model introduced by Avestimehr, Diggavi and Tse in [5]. For this special case, several unicast $[6,7,8]$, and multicast [4] algorithms were given. These approaches rely on the layered structure of the model, which is a strong restriction compared to the NCCP. Former randomized algorithms for the NCCP such as [9] have a lower bound on the required field size which depends on the size of the network and the number of terminals. We eliminate the first factor and present randomized algorithms for both the unicast and multicast cases over any field of size greater than the number of terminals. Our approach relies on the idea of a simple deterministic algorithm for the NCCP, constructing a solution for the multicast problem from solutions of the unicast special case.

We also define a related new problem, called 'fixable pairs problem'. We give a sufficient condition for a subset of coding coefficients that can be fixed arbitrarily to nonzero values, such that the remaining coefficients can be chosen properly to attain a feasible network code. We present applications of this model, and give necessary and sufficient conditions for the solvability of network coding problems in heterogenous networks.

The rest of the paper is organized as follows. In Section 2 definitions of network coding and its investigated special cases are given. In Section 3 we present deterministic and randomized algorithms for the NCCP. In Section 4 we discuss the problem of fixable pairs and present some applications in heterogenous networks.

\section{Problem formulation}

Definition 1. Let $\mathbb{F}_{q}$ be a finite field of size $q$ and let $\mathbb{F}_{q}^{k}$ denote the $k$ dimensional vector space over $\mathbb{F}_{q}$ and let $\mathbf{e}_{i}$ denote the $i$ th unit vector in $\mathbb{F}_{q}^{k}$, $1 \leq i \leq k$. For a set $W$ of vectors in $\mathbb{F}_{q}^{k},\langle W\rangle$ denotes the linear subspace spanned by $W$. Let $\mathbf{M}=\left(M_{1}, M_{2}, \ldots, M_{k}\right), M_{i} \in \mathbb{F}_{q}, 1 \leq i \leq k$ be an ordered set of $k$ messages.

Let $D=(V, A)$ be an acyclic directed graph with node and arc set $V$ and $A$, respectively, with a single source node $s$, from which the messages are sent, and a set of nodes $T \subseteq V-s$ called terminals where the messages are sent to. Without loss of generality we may assume that $s$ has $k$ leaving $\operatorname{arcs} a_{1}, \ldots, a_{k}$. A linear network code of $k$ messages on $D$ over $\mathbb{F}_{q}$ is a mapping $\mathbf{c}: A \rightarrow \mathbb{F}_{q}^{k}$ satisfying $\mathbf{c}\left(a_{i}\right)=\mathbf{e}_{i}$ which fulfills the linear combination property:

$$
\mathbf{c}(u v)=\sum_{w u \in A} \alpha(w u, u v) \mathbf{c}(w u)
$$

where $\alpha(w u, u v) \in \mathbb{F}_{q}$. Coefficients $\alpha(w u, u v)$ are the local coefficients of the network code and function $\mathbf{c}$ denotes the global coefficients. That is, on $\operatorname{arc} a$ message $\mathbf{c}(a) \cdot \mathbf{M}$ is sent. We will use the notation $\langle\mathbf{c}, u\rangle=\langle\{\mathbf{c}(w u) \mid w u \in A\}\rangle$. 
For a linear network code $\mathbf{c}$, a node $v$ can decode (or receives) message $M_{i}$, if $\mathbf{e}_{i} \in\langle\mathbf{c}, v\rangle$. A network code is feasible if every node $t$ in $T$ can decode every message $M_{i}, 1 \leq i \leq k$. The network coding problem given by parameters $D, s, T, k, q$ is to construct a feasible network code of $k$ messages in $D$ over $\mathbb{F}_{q}$. Note that global coefficients can be determined from local coefficients, hence feasibility of the latter can be defined accordingly. If $|T|=1$, the problem is called a unicast problem, while the general case is called multicast. The latter can be regarded as a network code construction which is simultaneously feasible for $|T|$ unicast problems.

Definition 2. Let $L \subseteq A \times A$ be the set of consecutive pairs of arcs: $L=$ $\{(w u, u v) \mid w, u, v \in V, w u, u v \in A\}$. For the sake of shortness members of $L$ are called pairs. The local coefficients of a network code form a mapping on the pairs: $\alpha: L \rightarrow \mathbb{F}_{q}$. For a subset of pairs $M \subseteq L$, a mapping $\alpha_{0}: M \rightarrow \mathbb{F}_{q}$ is extendable, if there exist local coefficients $\alpha$ of a feasible network code such that $\alpha=\alpha_{0}$ on $M$. Given a network coding problem with a subset of pairs $M \subseteq L$ with a mapping $\alpha_{0}: M \rightarrow \mathbb{F}_{q}$, the network code completion problem is to decide whether $\alpha_{0}$ is extendable.

Definition 3. We say that $M \subseteq L$ is fixable if any nonzero-valued mapping $\alpha_{0}: M \rightarrow \mathbb{F}_{q}-\{0\}$ is extendable. The fixable pairs problem is to decide whether a given set $M$ is fixable or not. For a pair $\ell=(w u, u v) \in L, w u$ and $u v$ are the first and second arcs of the pair, respectively, and $u$ is the central node of the pair. Two pairs $\ell_{1}$ and $\ell_{2}$ are consecutive if the second arc of $\ell_{1}$ is the first arc of $\ell_{2}$. A path contains a pair, if it contains both of its arcs. For a subset of pairs $M \subseteq L$, a node is $M$-influenced if it is the central node of a pair in $M$. A set of paths is $M$-independent if they are pairwise arc-disjoint and any $M$-influenced node is contained by at most one of them.

We use two well-known technical statements several times in this paper. Their proof can be found for example in [10].

Claim 4. Let vectors $\mathbf{v}_{1}, \ldots, \mathbf{v}_{k} \in \mathbb{F}_{q}^{k}$ form a basis of $\mathbb{F}_{q}^{k}$ and let $\mathbf{v} \in \mathbb{F}_{q}^{k}$. Then

- there is at most one value $\alpha \in \mathbb{F}_{q}$ not satisfying that the set $\left\{\mathbf{v}_{1}^{\prime}=\mathbf{v}_{1}+\right.$ $\alpha \mathbf{v}\} \cup\left\{\mathbf{v}_{i}\right\}_{i=2}^{k}$ is also a basis,

- there is at most one value $\beta \in \mathbb{F}_{q}$ not satisfying that the set $\left\{\mathbf{v}_{1}^{\prime}=\beta \mathbf{v}_{1}+\right.$ $\mathbf{v}\} \cup\left\{\mathbf{v}_{i}\right\}_{i=2}^{k}$ is also a basis.

\section{Network Coding Completion Problem}

The multicast NCCP is equivalent to determining the simultaneous max rank completion of the transfer matrices, and if the field size is greater than the number of matrices given, then the matrices have a simultaneous max rank completion as proved in $[9,3]$. This result can be reformulated as follows. 
Theorem 5. [3] If $q>|T|$, a mapping is extendable over $\mathbb{F}_{q}$ if and only if for every $t \in T$ it is extendable for the one-element terminal set $\{t\}$. Such an extension can be found in polynomial time.

We give another, simple proof for this theorem. We use the polynomial time algorithm of [3] for the unicast case as a subroutine.

Proof of Theorem 5. For a terminal $t \in T$, let $\alpha_{t}$ denote the extension of $\alpha_{0}$ which is feasible for $t$ and let $\mathbf{c}_{t}: A \rightarrow \mathbb{F}_{q}^{k}$ denote the corresponding global coefficients. We start by defining $\alpha(\ell)=\alpha_{0}(\ell)$ for each $\ell \in M$. Let $\ell_{1}, \ldots, \ell_{p}$ be an arbitrary order of the pairs in $L \backslash M$. We will determine a value $\alpha\left(\ell_{i}\right)$ for each $\ell_{i}$ in this order maintaining that the following mappings $\alpha_{t}^{i}$ are feasible for every $t$.

$$
\alpha_{t}^{i}(\ell)= \begin{cases}\alpha(\ell) & \text { if } \ell \in M \cup\left\{\ell_{1}, \ldots, \ell_{i}\right\} \\ \alpha_{t}(\ell) & \text { otherwise }\end{cases}
$$

To show the existence of an appropriate $\alpha\left(\ell_{i}\right)$ we prove some lemmas.

Lemma 6. Let $\alpha, \mathbf{c}$ denote the local and global coding coefficients of a network code, respectively. By altering a local coefficient $\alpha(u v, v w)$ to $\alpha^{\prime}(u v, v w)=$ $\alpha(u v, v w)+\beta$, the new global coefficients have the form $\mathbf{c}^{\prime}(a)=\mathbf{c}(a)+\delta_{a} \beta \mathbf{c}(u v)$ with an appropriate value $\delta_{a} \in \mathbb{F}_{q}$ on every arc $a \in A$.

Proof. We prove by induction on the topological order of the tails of the arcs. If the tail of an arc is earlier in the order than $v$, then $\mathbf{c}^{\prime}$ remains $\mathbf{c}$ and the claim clearly holds. For arcs leaving $v$, the only arc where $\mathbf{c}$ changes is arc $v w$, and the claim is again clear. Suppose that the claim holds for every arc with tail before $z \in V$ and let $z x \in A$ be an arc. From the linear combination property we have $\mathbf{c}^{\prime}(z x)=\sum_{y z \in A} \alpha(y z, z x) \mathbf{c}^{\prime}(y z)=\sum_{y z \in A} \alpha(y z, z x)\left(\mathbf{c}(y z)+\delta_{y z} \beta \mathbf{c}(u v)\right)$ $=\mathbf{c}(z x)+\beta \mathbf{c}(u v) \sum_{y z \in A} \alpha(y z, z x) \delta_{y z}$, which proves this lemma.

Lemma 7. Let vectors $\mathbf{v}_{1}, \ldots, \mathbf{v}_{k} \in \mathbb{F}_{q}^{k}$ form a basis of $\mathbb{F}_{q}^{k}$ and let $\mathbf{v} \in \mathbb{F}_{q}^{k}$, $\delta_{1}, \ldots, \delta_{k} \in \mathbb{F}_{q}$. Then there is at most one value $\beta \in \mathbb{F}_{q}$ satisfying that $\left\{\mathbf{v}_{i}^{\prime}=\right.$ $\left.\mathbf{v}_{i}+\delta_{i} \beta \mathbf{v}\right\}_{i=1}^{k}$ is not a basis.

Proof. If every $\delta_{i}$ is zero then the statement is obvious, so without loss of generality we can assume that $\delta_{1} \neq 0$. By subtracting $\left(\delta_{i} / \delta_{1}\right) \mathbf{v}_{1}^{\prime}$ from each $\mathbf{v}_{i}$ $(i=2, \ldots, k)$, we get that vectors $\left\{\mathbf{v}_{i}^{\prime}\right\}_{i=1}^{k}$ form a basis if and only if $\left\{\mathbf{v}_{1}+\right.$ $\left.\delta_{1} \beta \mathbf{v}\right\} \cup\left\{\mathbf{v}_{i}-\left(\delta_{i} / \delta_{1}\right) \mathbf{v}_{1}\right\}_{i=2}^{k}$ does. Since $\left\{\mathbf{v}_{1}\right\} \cup\left\{\mathbf{v}_{i}-\left(\delta_{i} / \delta_{1}\right) \mathbf{v}_{1}\right\}_{i=2}^{k}$ is a basis, the lemma follows from Claim 4.

Let $\mathbf{c}_{t}^{i}$ denote global coefficients corresponding to $\alpha_{t}^{i}$.

Lemma 8. Suppose that the values $\alpha\left(\ell_{1}\right), \ldots, \alpha\left(\ell_{i-1}\right)$ are chosen such that $\mathbf{c}_{t}^{i-1}$ is feasible for $t$. Then for each $t$, there is at most one choice of $\alpha\left(\ell_{i}\right)$ such that $\mathbf{c}_{t}^{i}$ is not feasible for $t$.

Proof. Since $\mathbf{c}_{t}^{i-1}$ is feasible, there is a $k$-element arc set $B_{t}=\left\{b_{1}, \ldots, b_{k}\right\}$ entering $t$ on which the global coefficients of $\mathbf{c}_{t}^{i-1}$ form a basis. Let $\ell_{i}=(u v, v w)$. 
Mappings $\alpha_{t}^{i-1}$ and $\alpha_{t}^{i}$ differ in at most one value (on $\ell_{i}$ ), hence from Lemma 6 we get that global coefficients on arcs in $B_{t}$ have the following form: $\mathbf{c}_{t}^{i}\left(b_{j}\right)=$ $\mathbf{c}_{t}^{i-1}\left(b_{j}\right)+\delta_{b_{j}}\left(\alpha_{t}^{i}\left(l_{i}\right)-\alpha_{t}^{i-1}\left(l_{i}\right)\right) \mathbf{c}_{t}^{i-1}(u v)$. Lemma 7 says that there is at most one value of $\ell_{i}$ such that these vectors do not form a basis.

Since the size of the field is greater than $|T|$, indeed, a good value can be chosen for each $\alpha\left(\ell_{i}\right)$. If $i=p$ then for every $t$ we have $\alpha_{t}^{p}=\alpha$, and we maintained the feasibility. This completes the proof of Theorem 5 .

\section{Randomized Algorithm}

Let a multicast NCCP be given as described in Section 2. Kim and Médard [9] gave a lower bound on the probability of a random network code to be feasible over $\mathbb{F}_{q}$ in the model of $[5]$.

Lemma 9. [9] If $q>|T|$ and a mapping $\alpha_{0}: M \rightarrow \mathbb{F}_{q}$ has a feasible extension, then the probability that a random extension is feasible, is at least $\left(1-\frac{|T|}{q}\right)^{\left|A^{\prime}\right|} \geq$ $1-\frac{|T| \cdot\left|A^{\prime}\right|}{q}$, where $A^{\prime}$ is the subset of arcs which appear as a second arc in a pair in $L \backslash M$.

The idea of our Las Vegas algorithm is to first construct a random extension over a bigger field $\mathbb{F}_{q^{r}}$ of size $q^{r}$ such that $\mathbb{F}_{q}$ is a subfield of $\mathbb{F}_{q^{r}}$. From Lemma 8 we can deterministically modify it to get another extension over $\mathbb{F}_{q}$ if $q>|T|$. Let $\alpha$ be a random extension of $\alpha_{0}$ over $\mathbb{F}_{q^{r}}$. If we choose $r$ such that $q^{r}>2|T| \cdot\left|A^{\prime}\right|$, then from Lemma $9, \alpha$ is feasible with probability at least one half. If the extension is not feasible, we repeat generating other random extensions till success. The expectation of the number of extension generations is two. If $\alpha$ is feasible, the extension over $\mathbb{F}_{q}$ is constructed by choosing for all $\ell \in L \backslash M$ one-by-one a value $f$ from $\mathbb{F}_{q}$ such that $\alpha$, changed only on $\ell$ to $\alpha(\ell)=f$, remains a feasible network code.

Theorem 10. If $q>|T|$, and there exists a feasible extension over $\mathbb{F}_{q}$ then the algorithm finds one with probability one in polynomial expected running time.

Proof. We only need to show that a suitable value from $\mathbb{F}_{q}$ can be chosen for every pair $\ell \in L \backslash M$. In Lemma 6 we gave a formula, how the modification of a local coefficient influences global coefficients. Combined with Lemma 7, for each $t \in T$ we get that there is at most one value $f \in \mathbb{F}_{q^{r}}$ such that changing the value of $\alpha(\ell)$ to $f$ destroys feasibility to $t$. Hence in any subset $X \subseteq \mathbb{F}_{q^{r}}$ with $|X|>|T|$ there exists a value which preserves feasibility for every terminal simultaneously. Since $q>|T|$, subfield $\mathbb{F}_{q}$ is such a subset, which proves the theorem.

\section{Sufficient Condition for a fixable set}

In this section we give a sufficient condition for a subset $M$ of pairs to be fixable and present some applications in heterogenous networks. 


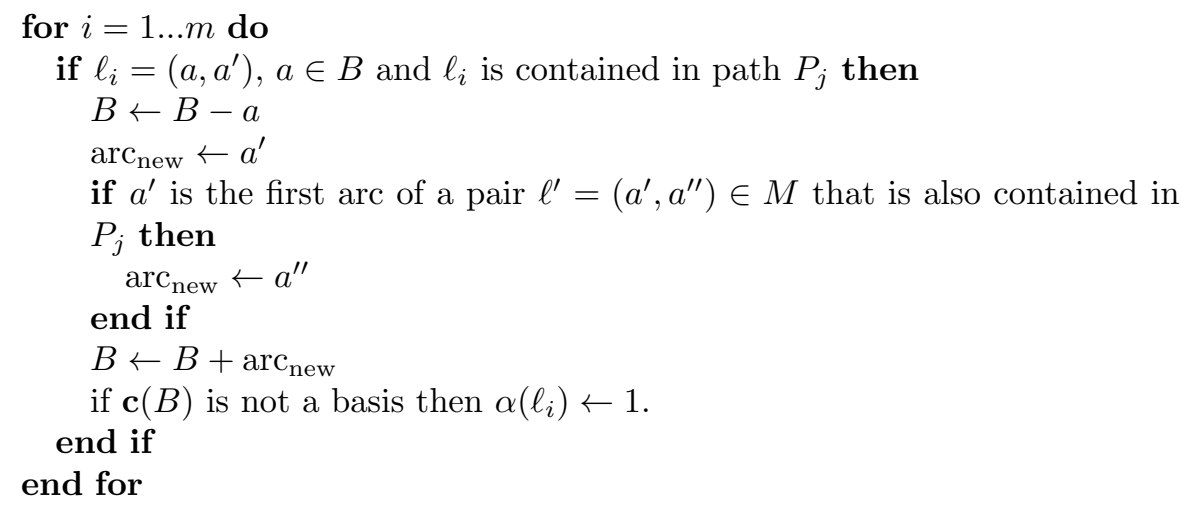

Figure 1: Exchanging an arc in $B$

Theorem 11. Let $D=(V, A)$ an acyclic directed graph and $T \subseteq V-s$ a terminal set having a feasible network code for $k$ messages over $\mathbb{F}_{q}$ with $q>|T|$, and let $M \subseteq L$ be a subset of pairs. If for every terminal $t \in T$ there exist $k M$-independent paths from $s$ to $t$, such that none of the paths contains two consecutive pairs in $M$, then $M$ is fixable.

PROOF. We follow similar ideas for the network code construction as the ones in [10] but instead of determining the global coefficients one-by-one in a topological order we determine the local coefficients in a special order. Let $\alpha_{0}$ be an arbitrary nonzero-valued mapping on $M$. From Theorem $5, M$ is extendable if and only if it is extendable for every one-element terminal set $\{t\}$. Let $t$ be an arbitrary given terminal in $T$. We choose $k M$-independent st-paths $P_{1}, \ldots, P_{k}$ such that no path contains two consecutive pairs in $M$. (This can be done by applying classical graph transformation techniques.) First we consider the extension $\alpha$ of $\alpha_{0}$ which is zero on $L \backslash M$. Let $\mathbf{c}$ denote the global coefficients corresponding to $\alpha$. If $\alpha$ alters during the algorithm then $\mathbf{c}$ is modified accordingly. Let us fix a topological order of the nodes and let $\ell_{1}, \ldots, \ell_{|L \backslash M|}$ be an order of the pairs in $L \backslash M$ according to the topological order of the heads of the second arcs. We determine the values of $\alpha$ on the pairs in this order and maintain a set of $\operatorname{arcs} B=\left\{b_{1}, \ldots, b_{k}\right\}$, such that $b_{i} \in P_{i}$, and $\mathbf{c}(B)=\left\{\mathbf{c}\left(b_{i}\right)\right\}_{i=1}^{k}$ forms a basis, and that $B$ finally contains only arcs entering $t$. First, let $B=\left\{a_{1}, \ldots, a_{k}\right\}$. If there is a pair of the form $\left(a_{i}, a\right)$ in $M$, where $a \in P_{i}$, then we replace $a_{i}$ with $a$ in $B$, see Figure 1 .

Claim 12. The modification of $\alpha\left(\ell_{i}\right)$ does not modify any arc in $B-\operatorname{arc}_{\text {new }}$.

Proof. The modification of $\alpha\left(\ell_{i}\right)$ influences an arc $e$ if and only if there is a path $a^{\prime}=e_{0}, e_{1}, e_{2}, \ldots, e_{z}=e$ such that $\alpha\left(e_{x}, e_{x+1}\right)$ is not zero for $0 \leq x<z$. Note that such a pair cannot be in $L \backslash M$ because these pairs have bigger index in our ordering, so their $\alpha$-values would be still zero. Hence $\left(e_{x}, e_{x+1}\right) \in M$ for each $0 \leq x<z$. Suppose that there exists such an arc $e$ which is in $B$ and is 
not contained in $P_{j}$. Since $P_{j}$ contains the head of $a^{\prime}$, which is the central node of $\left(a^{\prime}, e_{1}\right)$ and the paths are $M$-independent, $e_{1}$ cannot be in a path different from $P_{j}$, so $z \geq 2$. Hence both the tail and head of $e$ are different from the head of $a^{\prime}$. There was a point when $e$ got into $B$, let $\ell(e)$ be the pair that was being processed at that point. There are two cases: either $e$ is the second arc of $\ell(e)$ or $e$ is an arc following the second arc of $\ell(e)$. In both cases, the head of the second arc of $\ell(e)$ is reachable from the head of the second arc of $\ell_{i}$. From the choice of the order of processing the pairs, $\ell(e)$ should be processed later than $\ell_{i}$, which contradicts that $\ell(e)$ was processed earlier than $\ell_{i}$.

Claim 13. After processing a pair, $\mathbf{c}(B)$ form a basis of $\mathbb{F}_{q}^{k}$.

Proof. If $\alpha(\ell)$ remained 0, the claim clearly holds. Using Claim 12 we observe that we can apply Claim 4 , so only one value $\beta$ is wrong. Thus as zero was wrong, value 1 must be good.

Claim 14. After processing a pair $\ell$ in the algorithm, for every arc $b$ in $B$ one of the following hold:

- $b$ enters $t$,

- for the arc $b^{\prime}$ following $b$ on $P_{j}$ the pair $\left(b, b^{\prime}\right)$ is not in $M$.

Proof. Suppose that arc $b$ does not enter $t$ and $\left(b, b^{\prime}\right) \in M$. Let us take the step when $b$ got into $B$. From the choice of $P_{j}$, there are no consecutive pairs from $M$ on $P_{j}$, so $b$ cannot be the second arc of a pair in $M$ contained in $P_{j}$. Hence $\operatorname{arc}_{\text {new }}$ should have been $b^{\prime}$ instead of $b$.

From Claim 14 and the choice of the order of pairs the final set $B$ will only contain arcs entering $t$, which proves the theorem.

\section{Heterogenous networks}

Here we give a characterization for the network capacity including the case when some of the nodes are broadcasting. Let a network coding problem be given as described in Section 2. Suppose that a subset $W \subseteq V \backslash(T \cup\{s\})$ of intermediate nodes can only broadcast messages, that is, such a node sends the same message on each of its outgoing arc. The $W$-broadcasting network coding problem is to decide the existence of a network code where every node in $W$ broadcasts. To the best of our knowledge, there has been no characterization known on the existence of a feasible $W$-broadcasting network code. We say that a set of $s t$-paths is $W$-disjoint if the paths are pairwise arc-disjoint and each node in $W$ is contained in at most one of the paths. Note that the existence of $k W$-disjoint st-paths can be checked in polynomial time.

Theorem 15. Given a $W$-broadcasting network coding problem with $q>|T|$ and, there exists a feasible network code, if and only if for every $t \in T$ there are $k W$-disjoint st-paths. 
Proof. We are going to reduce the $W$-broadcasting problem to a special case of a fixable pairs problem. Let $D^{\prime}$ denote the graph attained from $D$ by expanding each node $w \in W$ into two new nodes $w_{i}$ and $w_{o}$ with a new arc $w_{i} w_{o}$ such that the incoming and outgoing arcs of $w$ become the incoming and outgoing arcs of $w_{i}$ and $w_{o}$, respectively. For a node $v \in V \backslash W$ let $v=v_{i}=v_{o}$. If there is a feasible $W$-broadcasting network code $\mathbf{c}$ on $D$, then it can be modified to be a feasible network code $\mathbf{c}^{\prime}$ on $D^{\prime}$ by setting for $u v \in A: \quad \mathbf{c}^{\prime}\left(u_{o} v_{i}\right)=\mathbf{c}(u v)$ and for $w \in W$ and for any $w v \in A$ we define $\mathbf{c}^{\prime}\left(w_{i} w_{o}\right)=\mathbf{c}(w v)$, this is legal as $w$ is a broadcasting node, consequently $\mathbf{c}(w v)$ is the same on every outgoing arc. Clearly, the existence of $k W$-disjoint st-paths in $D$ is equivalent with the existence of $k$ arc-disjoint st-paths in $D^{\prime}$. This gives that the conditions of the theorem are necessary. For the other direction, let $M$ be the following subset of pairs in $D^{\prime}: M=\left\{\left(w_{i} w_{o}, w_{o} v_{i}\right) \mid w \in W, w v \in A\right\}$. Note that $M$ does not contain consecutive pairs and since every central node of a pair in $M$ has in-degree one, $M$-independentness follows from arc-disjointness. Applying Theorem 11 we get that if there exist $W$-disjoint paths in $D$ then $M$ is fixable in $D^{\prime}$ and so we can take a feasible extension of the constant 1-valued mapping on $M$. Let $\mathbf{c}^{\prime}$ denote the global coefficients of the network code. One can easily get the global coefficients of a feasible network code on $D$ by contracting arcs in $D^{\prime}$ of the form $w_{i} w_{o}, w \in W$.

The fixable pairs problem can similarly model restrictions on incoming messages, if each node in a subset $W$ can only receive a fixed nowhere zero linear combination of their incoming messages. This can also be handled applying Theorem 11 on the auxiliary graph $D^{\prime}$ of Theorem 15 , by fixing for each $w \in W$ the local coefficient on a pair of the form $\left(u_{o} w_{i}, w_{i} w_{o}\right)$ to the corresponding value in the fixed linear combination.

Theorem 16. Let a network coding problem be given with $q>|T|$ and a subset $W \subseteq V$ such that every node in $W$ only receives a fixed nowhere zero linear combination of its incoming messages. There exists a feasible network code if and only if for every $t \in T$ there are $k W$-disjoint st-paths.

An important application is when some intermediate nodes only receive the XOR of their incoming messages (we assume that messages are from a finite field of size $2^{d}$ represented by $d$ bits). The XOR of the incoming messages can be regarded as the sum over the finite filed, and for each $w \in W$, every local coefficient on a pair of the form $\left(u_{o} w_{i}, w_{i} w_{o}\right)$ is fixed to 1 .

\section{Conclusion}

In this paper, different versions of the network code completion problem were considered. We presented a new, simple, algorithmic proof for the minmax-type property of the multicast NCCP. We also gave randomized algorithm over any field $\mathbb{F}_{q}$ with $q>|T|$ for both the unicast and multicast cases. We proposed the fixable pair problem, gave a sufficient condition and showed some applications to networks with varying node transmission properties. It remained open to give an exact characterization for a subset of pairs to be fixable. 


\section{Acknowledgment}

The authors received grants (no. CNK 77780 and no. K 109240) from the National Development Agency of Hungary, based on a source from the Research and Technology Innovation Fund.

[1] R. Ahlswede, N. Cai, S.-Y. R. Li, R. W. Yeung, Network information flow, IEEE Transactions on Information Theory 46 (4) (2000) 1204-1216.

[2] R. W. Yeung, S.-Y. R. Li, N. Cai, Z. Zhang, Network Coding Theory - Part I: Single Source, Vol. 2 of Foundations and Trends in Communications and Information Theory, now Publishers Inc., 2005.

[3] N. J. A. Harvey, D. R. Karger, K. Murota, Deterministic network coding by matrix completion, in: SODA, SIAM, 2005, pp. 489-498.

[4] J. Ebrahimi, C. Fragouli, Multicasting algorithms for deterministic networks, in: IEEE Information Theory Workshop (ITW), 2010. doi:10.1109/itwksps.2010.5503221.

[5] A. S. Avestimehr, S. N. Diggavi, D. N. C. Tse, A deterministic approach to wireless relay networks, in: Proceedings of the 45th annual Allerton conference on Communication, control, and computing, Allerton'07, 2007, pp. $721-728$.

[6] S. M. S. Tabatabaei Yazdi, S. A. Savari, A max-flow/min-cut algorithm for a class of wireless networks, in: Proceedings of the Twenty-First Annual ACM-SIAM Symposium on Discrete Algorithms.

[7] A. Amaudruz, C. Fragouli, Combinatorial algorithms for wireless information flow, in: Proceedings of the twentieth Annual ACM-SIAM Symposium on Discrete Algorithms, SODA '09, 2009, pp. 555-564.

[8] M. X. Goemans, S. Iwata, R. Zenklusen, An algorithmic framework for wireless information flow, in: Proceedings of the 47th annual Allerton conference on Communication, control, and computing, Allerton'09, 2009, pp. 294-300.

[9] M. Kim, M. Médard, Algebraic network coding approach to deterministic wireless relay networks, in: Proceedings of the 48th annual Allerton conference on Communication, control, and computing, Allerton'10, 2010, pp. $1518-1525$.

[10] S. Jaggi, P. Sanders, P. A. Chou, M. Effros, S. Egner, K. Jain, L. M. G. M. Tolhuizen, Polynomial time algorithms for multicast network code construction., IEEE Transactions on Information Theory (2005) 1973-1982. 